Check for updates

Cite this: Phys. Chem. Chem. Phys., 2020, 22, 12713

Received 23rd February 2020 Accepted 15th May 2020

DOI: $10.1039 / \mathrm{d} 0 \mathrm{cp} 01026 \mathrm{j}$

rsc.li/pccp

\title{
Hierarchically organized materials with ordered mesopores: adsorption isotherm and adsorption- induced deformation from small-angle scattering $\dagger$
}

\author{
Lukas Ludescher, (D) a Roland Morak, ${ }^{a}$ Stephan Braxmeier, ${ }^{b}$ Florian Putz, \\ Nicola Hüsing, (D) ${ }^{c}$ Gudrun Reichenauer ${ }^{b}$ and Oskar Paris (D) *a
}

\begin{abstract}
In situ small angle scattering is used to study the pore filling mechanism and the adsorption induced deformation of a silica sample with hierarchical porosity upon water adsorption. The high structural order of the cylindrical mesopores on a 2D hexagonal lattice allows obtaining adsorption induced strains from the shift of the corresponding Bragg peaks measured by in situ small-angle X-ray scattering (SAXS). However, apparent strains due to scattering contrast induced changes of the Bragg peak shapes emerge in SAXS. In contrast, small-angle neutron scattering (SANS) allows determining the real adsorption induced strains by employing a $\mathrm{H}_{2} \mathrm{O} / \mathrm{D}_{2} \mathrm{O}$ adsorbate with net coherent scattering length density of zero. This allows separating the apparent strains from the real strains experimentally and comparing them with strains obtained from model calculations of the SAXS intensity. It is shown that the apparent strains cannot be described at all by a simple mesopore model of film growth and capillary condensation. A hierarchical model taking the scattering of the micropores and the outer surface of the mesoporous struts in the hierarchically porous sample properly into account, together with a modified mesopore filling mechanism based on a corona model, leads to satisfactory description of both, the adsorption isotherm and the measured apparent strains as derived by SAXS.
\end{abstract}

\section{Introduction}

Nanoporous materials are used for a variety of applications including separation technologies and catalysis, gas- and energy storage, as well as sensors and actuators. ${ }^{1-4}$ Detailed information on the pore space characteristics of such materials is usually obtained from gas adsorption isotherms, ${ }^{5}$ supported by imaging methods such as scanning- or transmission electron microscopy, ${ }^{6}$ and by small-angle X-ray scattering (SAXS). ${ }^{7,8}$ Although 3D imaging techniques using X-rays and electrons are developing quickly, micropores and small mesopores below $10 \mathrm{~nm}$ are still hardly accessible to these methods, particularly in highly disordered nanoporous systems. In this regime, SAXS has proven a powerful method to characterize surface area and porosity $^{9,10}$ as well as size and shape of nanopores in detail. ${ }^{11,12}$ Moreover, SAXS allows studying the structure of host species within the pore space in situ during, e.g., gas adsorption at the nanopore surfaces ${ }^{12,13}$ gas-liquid- or liquid-solid phase transitions

\footnotetext{
${ }^{a}$ Institute of Physics, Montanuniversität Leoben, Franz-Josef Strasse 18, 8700, Leoben, Austria. E-mail: oskar.paris@unileoben.ac.at

${ }^{b}$ Bavarian Center for Applied Energy Research, Magdalene-Schoch-Str. 3, 97074 Wuerzburg, Germany

'Chemistry and Physics of Materials, Paris Lodron University Salzburg, Jakob-Haringer Str. 2a, 5020 Salzburg, Austria

$\dagger$ Electronic supplementary information (ESI) available. See DOI: 10.1039/d0cp01026j
}

in confinement, ${ }^{14}$ or ion arrangement in electrical double layers during charging. ${ }^{15}$

However, the analysis of the SAXS data is usually not straightforward since at least three phases (i.e., pores, solid host, and at least one liquid- or solid-like guest phase) are present. One of the assumptions often made is that the structure of the porous material does not change upon the uptake or phase-change of the guest phase. In other words, the structure of the "empty" porous material is determined first, and the changes observed in the SAXS signal are assumed exclusively to result from the occupation of the pore space by the guest phases and by their rearrangement. This assumption is however seldom met in reality, since the interaction of the guest phase with the solid pore walls leads to volume changes of the pore space, and potentially also to volume changes of the solid phase ranging from some fractions of percent ${ }^{16,17}$ to 50 vol\%. ${ }^{18,19}$ Indeed, adsorption induced deformation has been a topic of interest for a long time, ${ }^{20,21}$ but also volume changes induced by liquid-solid phase transitions ${ }^{22}$ or by electrosorption of ions in electrical double layers ${ }^{23-27}$ have both, scientific and application related relevance. In particular, the adsorption induced deformation observed as a function of relative gas pressure along an adsorption isotherm has been investigated in numerous experimental studies, ${ }^{28-30}$ and quite detailed theoretical descriptions based on fluid thermodynamics in confinement, coupled with solid mechanics have been developed, 
both for highly ordered as well as for disordered porous materials. $^{31-36}$ This allowed detailed modeling of adsorption induced deformation of cylindrical mesopores without ${ }^{34}$ and with microporous walls. ${ }^{37}$ This synergistic combination of experiments and theoretical modeling was possible by using hierarchical silica model materials comprising macroscopic samples with three hierarchical pore levels, i.e., macropores, mesopores and micropores, allowing the adsorption induced deformation to be measured using in situ dilatometry. ${ }^{18}$ The mesopores in these materials consist of cylinders with narrow diameter distribution, arranged on a hexagonal pore lattice, similar to the well-known SBA15 material. $^{38}$ This leads to Bragg reflections in SAXS measurements, and the adsorption induced deformation of the pore lattice was assumed to be directly accessible from the shift of the Bragg peaks. $^{30,39}$ However, considerable differences between adsorption strains from SAXS and dilatometry on the same samples were detected, ${ }^{40}$ which are not consistent with the predictions of the theoretical models for radial mesopore deformation measured by SAXS, and a combination of radial and axial deformation measured by dilatometry. ${ }^{34}$

It was already recognized earlier that the filling of the mesopore space in SBA-15 or MCM- $41^{41}$ can lead to so called "apparent strains" when analyzing the Bragg reflections from SAXS that are superimposing the actual deformation of the materials' backbone. These apparent effects are due to subtle peak shifts as a consequence of intensity changes during pore filling with the adsorbate. ${ }^{42}$ For this reason, small-angle neutron scattering (SANS) using water as an adsorbate with the scattering length density tuned to zero by a proper $\mathrm{H}_{2} \mathrm{O} / \mathrm{D}_{2} \mathrm{O}$ isotope mixture was recently employed. ${ }^{16}$ Hence, no intensity changes due to the scattering contrast, but only due to adsorption induced volume changes affect the scattering pattern, thus allowing the determination of the "real" adsorption induced deformation. ${ }^{17}$ Yet, the application of SANS is limited by several factors, such as limited availability of neutrons and limited resolution. Therefore, the determination of adsorption induced deformation using SAXS would be desirable, since such measurements can easily be performed using laboratory SAXS instruments with potentially considerably better strain resolution. This requires however the detailed modeling of the SAXS data, enabling the separation of "real" and "apparent" adsorption induced strains.

Here we develop a comprehensive model to simulate SAXS data from hierarchical silica with disordered micropores and ordered cylindrical mesopores during the adsorption of water. The model fully considers the three-phase nature of the system and predicts the SAXS profiles as a function of relative water pressure. Since we assume an infinitely stiff porous system in the model, the peak shifts deduced from the simulations are solely due to the apparent strains. These simulated apparent strains are then compared to apparent experimental strain data from a combined SAXS/SANS experiment.

\section{Materials and methods}

The investigated sample is a silica sample with hierarchical porosity (Fig. 1a). Materials synthesis was described in detail in a previous paper, ${ }^{17}$ and the sample was calcined at $500{ }^{\circ} \mathrm{C}$ to effectively remove organic residues from synthesis while limiting sintering, thus revealing micropores in the mesopore walls. ${ }^{43}$ Subsequently, the sample was aged at $74 \%$ relative humidity and $50{ }^{\circ} \mathrm{C}$ for 3 weeks to prevent reversible changes of the porous silica upon water adsorption. ${ }^{17}$ The sample was characterized by scanning electron microscopy (SEM) as well as with nitrogen adsorption as described in detail in ref. 17.

In situ small-angle neutron scattering (SANS) measurements were conducted at the SANS 1 instrument ${ }^{44}$ at the Heinz MaierLeibnitz Zentrum (MLZ, Garching near Munich, Germany), using a custom-built vapor dosing system ${ }^{16}$ connected to the sample cell. The adsorbate was a mixture of $91.95 \mathrm{wt} \%$ water and $8.05 \mathrm{wt} \%$ heavy water, chosen to obtain a net scattering length density of zero for neutrons (Z-SLD water). The relative pressure of the adsorbate in the sample cell was adjusted incrementally until equilibrium was reached for each measurement point and was kept constant during SANS data collection. The sample temperature was kept constant at 290.15 K. Details of the SANS experiments and data treatment are given in ref. 16.

In situ small-angle X-ray scattering (SAXS) experiments employing a similar vapor dosing setup and sample cell were conducted in-house using a laboratory instrument (NanoStar, Bruker AXS, Karlsruhe), equipped with a microfocus X-ray source with a copper anode. The beam was monochromatized to the $\mathrm{Cu}-\mathrm{K}_{\alpha}$ line by cross-coupled Göbel mirrors and collimated with two $300 \mu \mathrm{m}$ diameter scatterless pinholes. SAXS patterns were collected with a Vantec 2000 detector at a sample-detector distance of $72 \mathrm{~cm}$. SAXS measurement time was $45 \mathrm{~min}$ for each pressure point. The adsorptive was an identical $\mathrm{H}_{2} \mathrm{O} / \mathrm{D}_{2} \mathrm{O}$ mixture as for the neutrons at the same sample temperature of $290.15 \mathrm{~K}$.

SAXS and the SANS experiments were performed on the identical sample, and despite of the different illuminated sample areas due to different beam cross sections (neutron beam: $5 \mathrm{~mm}$, $\mathrm{X}$-ray beam $\approx 0.3 \mathrm{~mm}$ ), they were absolute identical. While SANS data were normalized to absolute units, SAXS measurements are given in arbitrary units here.

\section{Experimental results}

Fig. 1a shows SEM images of the meso- and macropore structure of the hierarchically porous silica sample. On the macropore scale, the monolithic sample consists of a network of interconnected struts with a length of roughly one micron and a thickness of 200-400 nm. Each strut contains well aligned parallel cylindrical mesopores. SANS (Fig. 1b) and SAXS (Fig. 1c) profiles confirm the ordered arrangement of the mesopores on a $2 \mathrm{D}$ hexagonal lattice similar to SBA-15. ${ }^{38}$ The resolution of the SANS data is not as good as the one of the SAXS data because of limited SANS detector resolution and broad wavelength spread from the neutron velocity selector $(10 \%)$, leading to considerable peak broadening. Scattering data for the sample are shown for the completely empty state $\left(p / p_{0}=0\right)$ and for a high relative pressure close to the bulk water saturation pressure, such that the microand mesopores are completely filled, while the macropores are 
a)

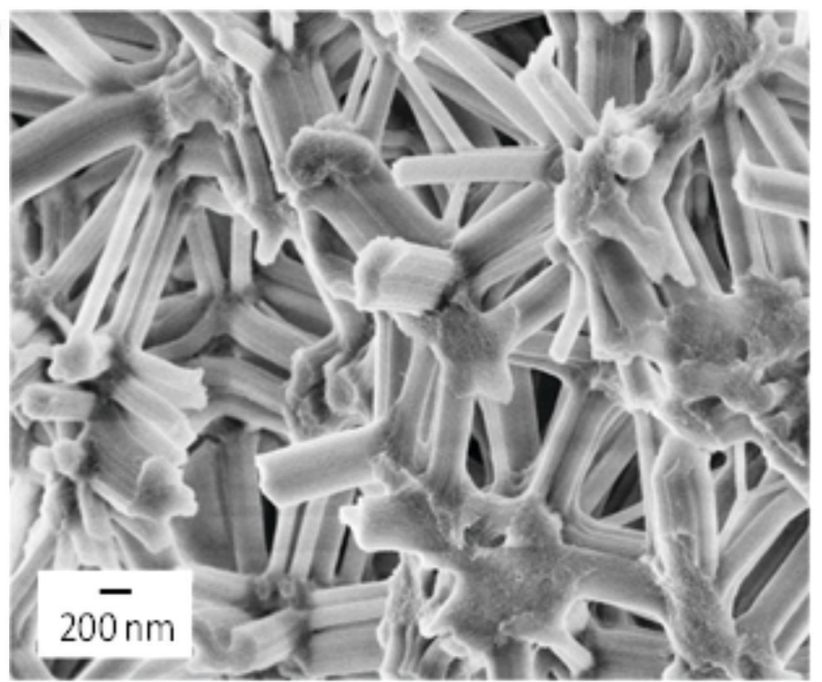

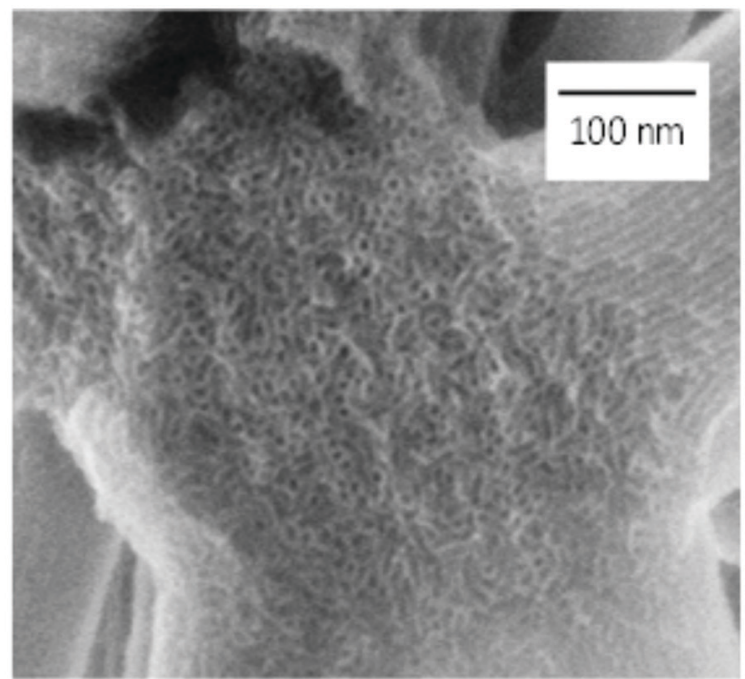

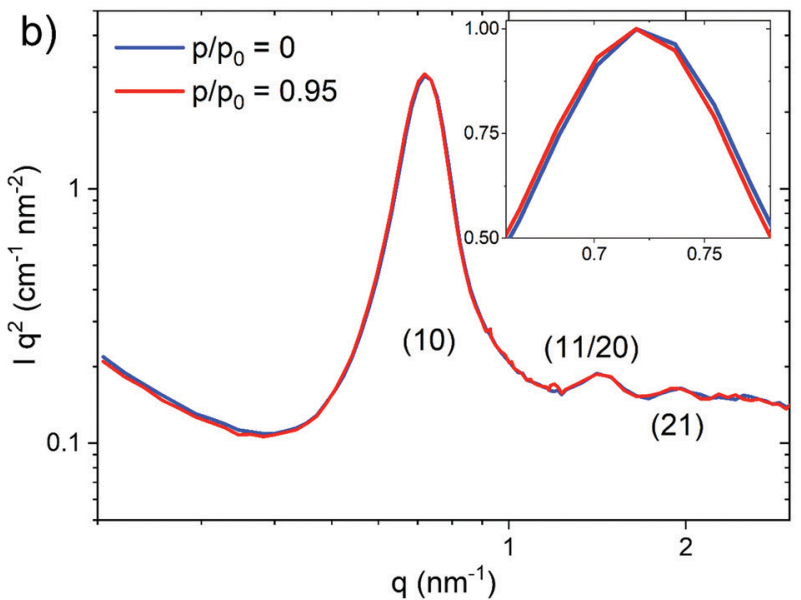

c)

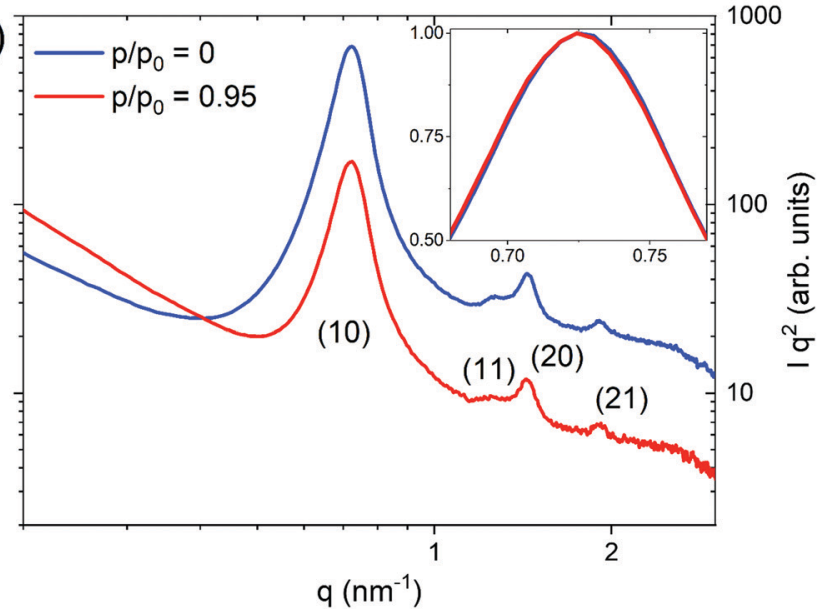

Fig. 1 (a) SEM images (taken from ref. 17) of the macroporous structure of the sample consisting of interconnected struts (left), where each strut comprises an array of ordered cylindrical mesopores (right). In situ SANS (b) and in situ SAXS (c) profiles in a Kratky plot representation for the empty sample (blue) and for the micro- and mesopores filled with neutron Z-SLD water (red). The numbers given in brackets are the Miller indices of a 2D hexagonal pore lattice. The insets show the normalized (10) peaks to visualize the peak shifts.

still empty. For both datasets a constant background scattering term was subtracted. For the SANS experiment (Fig. 1b), almost no intensity changes are observed between the empty and the filled sample as expected for Z-SLD water. For the SAXS experiment (Fig. 1c) however, a large drop in the scattering intensity occurs at large values of the scattering vector $q$ for the filled sample, while at the same time, the intensity at small $q$ is increased. This effect is due to the changed electron density (empty versus water-filled micro and mesopores) in the sample. Interestingly, the complete micro- and mesopore filling does not simply result in an overall shift of the SAXS curves, which is a consequence of the hierarchical structure of the sample. The scattering at small $q$ is dominated by the surface scattering of the whole strut. Since the overall electron density of the strut increases upon filling, its scattering contrast versus the empty macropores increases, leading to an increase of the intensity with filling. In the intermediate and large $q$-region, however, the scattering contrast is determined by the electron density difference between the meso-/micropores and the silica matrix. This scattering contrast decreases upon pore filling, thus leading to an intensity decrease. More generally speaking, with increasing relative pressure, the SAXS intensity profile is strongly affected by the adsorption of water molecules in the micropores and at the mesopore walls, and eventually by capillary condensation in the mesopores.

For the SANS data, a small peak shift to lower $q$ is clearly observed for the filled mesopores (visualized more clearly in the inset of Fig. 1b), suggesting adsorption induced expansion of the mesopore lattice. A slight peak shift is also observed in the SAXS data when normalized to the maximum of the (10) Bragg peak, although this shift is clearly smaller as compared to SANS (inset of the Fig. 1c). After quantitatively evaluating the peak position using the center of mass of the (10) peak, ${ }^{28}$ the mesopore lattice strain as a function of relative pressure is obtained from the relative peak shift, with the empty sample being the reference state. In Fig. $2 \mathrm{a}$ the strain isotherms using SAXS and SANS are shown for the adsorption branch of the sample. There are obviously clear differences between the SANS and the SAXS data. In particular, after an initial similar increase 

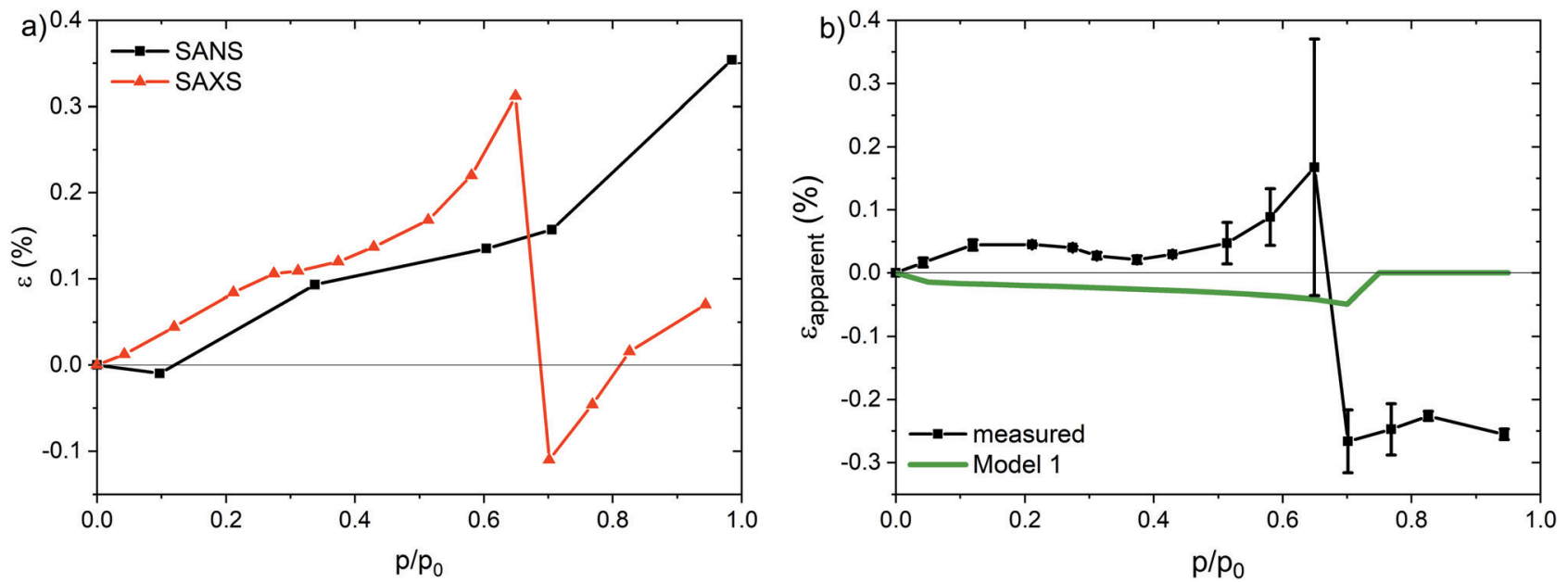

Fig. 2 (a) Experimental SANS (black symbols) and SAXS (red symbols) adsorption strain isotherms obtained from the shift of the (10) Bragg reflection during adsorption of water. In (b) the apparent strain isotherm (black symbols) is determined from the data in (a) using eqn (1). The green curve is the simulated apparent strain isotherm using a model for film growth and capillary condensation in a purely mesoporous system (Model 1 and Section 4.2)

of the strain with relative pressure, the SAXS strain isotherm shows a pronounced strain drop at capillary condensation, while the SANS strain isotherm exhibits only a slight dip. Beyond capillary condensation, the strain values in both isotherms increase again in a similar manner, but there remains a large offset between the SANS and the SAXS strain isotherms. A similar discrepancy was already observed earlier on a hierarchical silica sample, where the strain isotherm from SAXS was compared to a strain isotherm obtained from in situ dilatometry. ${ }^{40}$ However, theoretical models for cylindrical mesopores predict that the isotherms for axial and radial strains ${ }^{34,37}$ should approach the same value at $p / p_{0}=1$, which was also confirmed experimentally in ref. 17 by SANS using Z-SLD water.

From the strain isotherms in Fig. 2, we conclude that the strains extracted from the SAXS data are strongly affected by pore filling induced contrast changes, leading to apparent strain contributions. The isotherms obtained from SANS, however, can be taken as the true strains because of the use of Z-SLD water as adsorbate. Since both, the true strains (SANS) as well as the sum of apparent and true strains (SAXS) were measured on the same sample, the experimentally determined apparent strain is simply given by

$$
\varepsilon_{\text {apparent }}=\varepsilon_{\text {SAXS }}-\varepsilon_{\text {SANS }}
$$

Fig. 2b shows these "measured" apparent strains calculated using eqn (1), where data between the different $p / p_{0}$ measurement points for the SAXS and SANS data in Fig. 2a were linearly interpolated. We note that particularly close to capillary condensation this procedure leads to a large error due to an insufficient number of data points in this region. The measured apparent strain first increases slightly with relative pressure below capillary condensation and jumps to a large negative value above capillary condensation (Fig. 2b). For pressures above capillary condensation the apparent strain remains roughly constant as expected, because once the mesopores and micropores are completely filled with liquid, no significant intensity changes occur anymore. Yet, the apparent strain is not zero in this case as might be expected.

\section{Theoretical modeling}

Here we develop SAXS models aiming to quantitatively predict the measured apparent strains in Fig. $2 \mathrm{~b}$. Prass et al. ${ }^{42}$ have determined the apparent strains in cylindrical mesopores analytically and numerically in the relative pressure region close to capillary condensation. Here, we extend this approach aiming at deriving the apparent strains quantitatively for the whole range of relative pressures by calculating the SAXS patterns of a single mesoporous model-strut containing mesopores with microporous mesopore walls. Model 1 deals with the simplified case of liquid film formation in ordered mesoporous silica with no micropores, while Model 2 takes the micropores and their filling as well as a more realistic mesopore model into account.

\subsection{SAXS from cylindrical mesopores on a hexagonal lattice}

In the first step we set up a model to calculate the SAXS intensity for a purely mesoporous strut in its empty state. Since the length of the struts is much larger than the diameter of the mesopores (see Fig. 1a), a two-dimensional description is sufficient. The form factor of a circular mesopore cross-section is approximated by a step density model adapted from Zickler $e t a l .^{12}$

$$
F(q)=k\left((\alpha-1) R_{1}{ }^{2} \frac{2 J_{1}\left(q R_{1}\right)}{q R_{1}}+(\chi-\alpha) R_{2}{ }^{2} \frac{2 J_{1}\left(q R_{2}\right)}{q R_{2}}\right),
$$

with $J_{1}$ being the Bessel function of the first kind and first order and $k$ a constant. This model takes a rough surface (corona) of the mesopores into account, ${ }^{11,12}$ where $R_{1}$ and $R_{2}$ are the inner and outer radius of the corona, and

$$
\alpha=\rho_{\text {corona }} / \rho_{\mathrm{SiO}_{2}}
$$


is the ratio between the average electron densities of the corona and the silica mesopore wall, respectively. The parameter $\chi$ is defined by

$$
\chi=\left\{\begin{array}{ll}
0 & p / p_{0}<p_{\text {cap }} / p_{0} \\
\rho_{\mathrm{H}_{2} \mathrm{O}} / \rho_{\mathrm{SiO}_{2}} & p / p_{0} \geq p_{\text {cap }} / p_{0}
\end{array},\right.
$$

taking the jump in electron density within the mesopores into account when they spontaneously fill with liquid-like adsorbate upon capillary condensation, $p_{\text {cap }} / p_{0}$ being the relative pressure at capillary condensation.

For the empty sample we have $\chi=0$, and the two radii $R_{1}$ and $R_{2}$ as well as the volume fraction of silica in the corona, $\alpha=\alpha_{0}$, can be determined from the corresponding SAXS data of the empty sample by fitting the integrated Bragg peak intensities with eqn (2). ${ }^{12}$ From these parameters, an "equivalent mesopore radius" $R_{\mathrm{m}}=\sqrt{R_{2}^{2}+\left(1-\alpha_{0}\right)\left(R_{1}^{2}-R_{2}^{2}\right)}$ can be determined. ${ }^{45}$ The results of the fitting procedure (see ESI $\dagger$ for details) are given in Table 1, together with the center to center distance of the mesopores, $d=4 \pi /\left(\sqrt{3} q_{10}\right)$, obtained from the position $q_{10}$ of the (10) reflection. The total mesopore volume fraction (mesoporosity) is then calculated geometrically by $\phi_{\mathrm{m}}=(2 \pi / \sqrt{3})\left(R_{\mathrm{m}}^{2} / d^{2}\right)$, with the contribution of the corona $\phi_{\text {corona }}=(2 \pi / \sqrt{3})\left(\left(R_{\mathrm{m}}{ }^{2}-R_{2}{ }^{2}\right) / d^{2}\right)$ and the remaining volume fraction of mesopore space outside the corona, $\phi_{\mathrm{m}, 0}$.

To simulate the SAXS of the whole strut, we construct a single mesoporous strut of quadratic cross-section hosting an assembly of $30 \times 30$ hexagonally arranged cylindrical mesopores (Fig. 4a). It turns out that the specific cross-sectional shape of the strut is

Table 1 Mesopore parameters $R_{1}, R_{2}, \alpha_{0}, R_{\mathrm{m}}, d, \phi_{\mathrm{m}}, \phi_{\text {corona }}$ and $\phi_{\mathrm{m}, 0}$ obtained from SAXS

\begin{tabular}{llllllll}
\hline$R_{1}(\mathrm{~nm})$ & $R_{2}(\mathrm{~nm})$ & $\alpha_{0}$ & $R_{\mathrm{m}}(\mathrm{nm})$ & $d(\mathrm{~nm})$ & $\phi_{\mathrm{m}}$ & $\phi_{\text {corona }}$ & $\phi_{\mathrm{m}, 0}$ \\
\hline 3.81 & 2.39 & 0.5 & 3.19 & 10.1 & 0.36 & 0.16 & 0.20
\end{tabular}

not critical for the results, since the spherical average of different geometrical shapes results in the same surface scattering. The arrangement chosen corresponds roughly to a strut diameter of $350 \mathrm{~nm}$ which is in the same order of magnitude as in the real system (see Fig. 1a). The total scattered intensity from the mesoporous strut is then calculated numerically using the twodimensional Debye scattering equation. ${ }^{46}$

$$
I_{\mathrm{m}}(q)=\frac{1}{q} \sum_{i=1}^{n} \sum_{j=i}^{n} F_{i}(q) F_{j}(q) J_{0}\left(q d_{i j}\right)
$$

$F_{i}, F_{j}$ are the mesopore form factors calculated using eqn (2), $d_{i j}$ are the pairwise distances between the single mesopores, and $J_{0}$ is the Bessel-function of the first kind and order zero. The distances between neighboring mesopores were kept constant at the average lattice parameter value $d$ of the hexagonal pore lattice (Table 1), but some disorder in the lattice distances was taken into account by a random displacement of the pore centers from their ideal hexagonal lattice points according to a static Debye-Waller factor with a mean displacement of $0.09 \mathrm{~nm} .{ }^{11}$

\subsection{Model 1: film growth and capillary condensation in cylindrical mesopores}

In this model we assume perfect cylindrical mesopores with equivalent mesopore radius $R_{\mathrm{m}}$ and no micropores. The filling of the mesopores with water is modeled by assuming an increasing liquid film thickness on the mesopore walls with increasing relative pressure until the pores completely fill at capillary condensation. The form factor is described by eqn (2)-(4) with $R_{1}=R_{\mathrm{m}}, R_{2}=R_{\mathrm{film}}$ and $\alpha=\rho_{\mathrm{H}_{2} \mathrm{O}} / \rho_{\mathrm{SiO}_{2}}$. We note that the film thickness $t\left(p / p_{0}\right)=R_{\mathrm{m}}-R_{\mathrm{film}}\left(p / p_{0}\right)$ is the only pressure dependent quantity in this approach (Fig. 3a). The film thickness as a function of relative pressure was determined from the fit of the water adsorption isotherm (Fig. S1, ESI $\dagger$ ) using the Derjaguin-Broekhoff-de Boer (DBdB) approach for cylindrical mesopores. ${ }^{47,48}$ This procedure to determine the film thickness a)

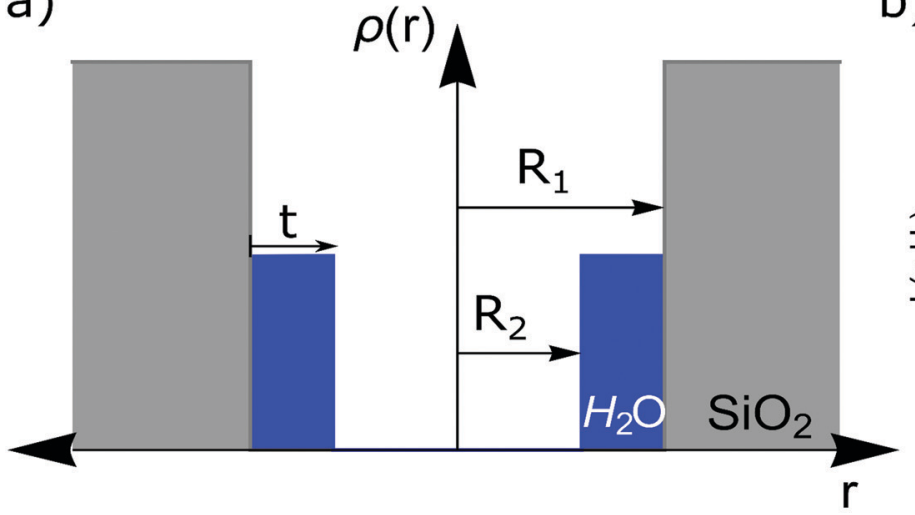

b)

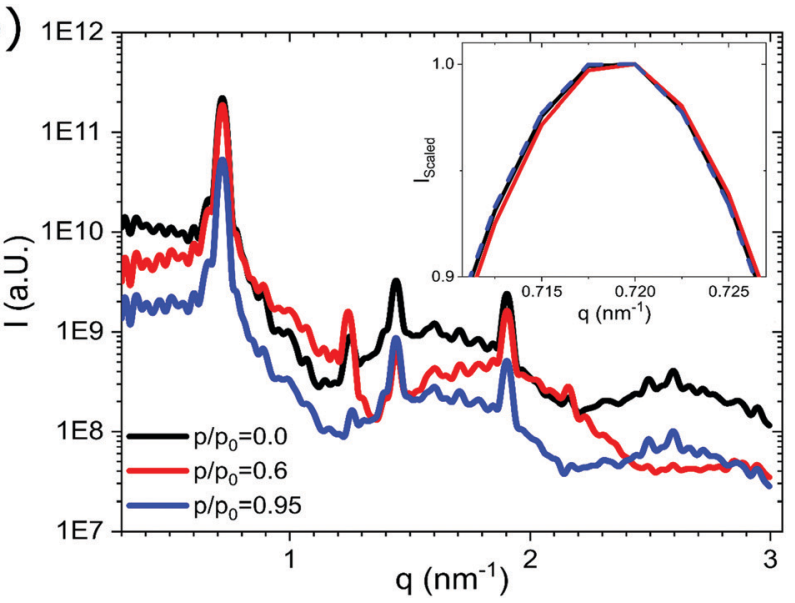

Fig. 3 (a) Step density model for mesopore filling by growth of a film with thickness $t=R_{1}-R_{2}, R_{1}$ being the equivalent mesopore radius $R_{\mathrm{m}}$. The vertical axis represents the electron densities of water (blue) and silica (grey). (b) Simulated SAXS patterns using eqn (3) for three different relative pressures corresponding to various film thicknesses ( $t=0 \mathrm{~nm}, t=0.34 \mathrm{~nm}$ and $t=R_{\mathrm{m}}$ ), with the inset depicting a zoom of the (10) peaks scaled to one for better visibility of the apparent strains. 
of cylindrical mesopores was already successfully applied to nitrogen $^{34,37}$ and pentane ${ }^{49}$ adsorption in silica, using corresponding reference isotherms for the silica-adsorbate interaction. Recently, we applied this approach also to water adsorption by combining the information from nitrogen and water adsorption isotherms to estimate the unknown water-silica interaction. ${ }^{17}$ The film-thickness as a function of relative pressure for the present sample is shown in Fig. S2 (ESI $\dagger$ ) for the equivalent mesopore radius $R_{\mathrm{m}}=3.19 \mathrm{~nm}$ taken from Table 1 . It should be noted that the film thickness also depends on the mesopore radius, and therefore, it needs to be calculated for every single pore in eqn (5). This also leads to different capillary condensation pressures for the different pore classes, thus covering the apparent strains in the region of capillary condensation as described by Prass et $a .^{42}$ To this end, the equivalent mesopore radii used to calculate the intensity with eqn (5) were normal distributed around the equivalent mesopore radius $R_{\mathrm{m}}$ (Table 1 ), with the width of the distribution estimated from the capillary condensation interval in the adsorption isotherm ${ }^{50}$ (see $\mathrm{ESI} \dagger$ for details).

Fig. $3 \mathrm{~b}$ shows the simulated SAXS patterns for the empty state (film thickness $t=0 \mathrm{~nm}$ ), for a film with thickness $t=0.34 \mathrm{~nm}$, and completely filled mesopores. A slight peak shift is observed in the inset of Fig. $3 \mathrm{~b}$ for the state with $t=0.34 \mathrm{~nm}$, confirming the presence of an apparent strain due to film formation. For the completely water filled sample, however, the peak position is exactly back to the situation at $p / p_{0}=0$. This is also expected from eqn (2), since $\chi=0$ for the empty sample and $\chi=\alpha$ for the filled sample after capillary condensation, leading to a simple reduction of the intensity by a factor $(\alpha-1)^{2}$ but no change in the shape of the SAXS curve. SAXS curves were simulated using eqn (5) for 20 distinct, evenly spaced relative pressures between 0 and 95\% using the film thickness shown in Fig. S2 (ESI $\dagger$ ). From the simulated SAXS patterns, the apparent strains were determined from the shift of the center of mass of the (10) Bragg peak in the Kratky representation. They are shown in Fig. 2b along with the corresponding experimental apparent strains. It is obvious that the model completely fails to predict the apparent strain isotherm of the investigated sample, both, in magnitude and direction. The simulated apparent strain is progressively negative with increasing pressure and jumps back to zero after capillary condensation. In contrast, the measured apparent strain is positive and increases until capillary condensation, where it drops to a large negative value and remains approximately constant there. From this observation we conclude that Model 1 is way too simple for micro-/mesoporous materials, suggesting that the filling of the corona and the micropores needs to be taken into account. We note however that this model might be applicable to purely mesoporous materials with reasonably smooth pore walls such as MCM- $41 .^{30}$

\subsection{Model 2: film growth and capillary condensation in cylindrical mesopores}

We start with the hierarchical pore model previously introduced by Gommes et al., ${ }^{10}$ with its three hierarchical contributions sketched in Fig. 4. The total SAXS intensity is assumed to be an
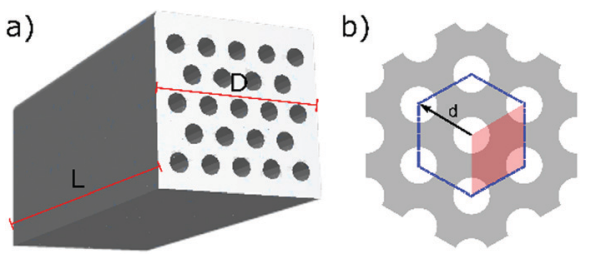

c)

Fig. 4 Individual contributions to the hierarchical pore model (eqn (6)). (a) Sketch of a single strut of length $L$ and side-length $D$; the number of mesopores used for the simulation $(30 \times 30)$ is not presented correctly here. (b) Top view of the mesopore level. The cylindrical mesopores are arranged in a 2-D hexagonal lattice with lattice parameter $d$. The unit cell of the pore lattice is highlighted by the opaque, red area, with a detailed zoom sketched in (c). The mean mesopore radius is $\bar{R}$, and micropores (assumed to be of spherical shape with radius $r_{\mu}$ ) are embedded in the silica mesopore walls.

incoherent superposition of scattering contributions from macro- (index M), meso- (index m) and micropores (index $\mu$ )

$$
I_{\mathrm{SAXS}}(q)=I_{\mathrm{M}}(q)+I_{\mathrm{m}}(q)+I_{\mu}(q)
$$

The first term in eqn (6) describes the scattering from the model strut, whereas the third term represents the scattering from micropores embedded in the mesopore walls. We note that the SAXS intensity was not determined in absolute units, therefore eqn (6) is only given up to an unknown multiplicative constant. We assume the macropore scattering $I_{\mathrm{M}}(q)$ to result from pure surface scattering of the strut, i.e., a $q^{-4}$ power law contribution according to Porod's law which changes in intensity due to the change of the average electron density within the strut during fluid adsorption ${ }^{10}$

$$
I_{\mathrm{M}}\left(q, p / p_{0}\right)=2 \pi\left(\left(1-\phi_{\mathrm{m}}\right)\left(1-\phi_{\mu}\right) \rho_{\mathrm{SiO}_{2}}+\phi_{\mathrm{H}_{2} \mathrm{O}}\left(p / p_{0}\right) \rho_{\mathrm{H}_{2} \mathrm{O}}\right)^{2} \frac{A_{\mathrm{g}}}{q^{4}},
$$

with $\phi_{\mathrm{m}}$ and $\phi_{\mu}$ being the mesopore and micropore volume fractions, respectively. $\rho_{\mathrm{SiO}_{2}}$ and $\rho_{\mathrm{H}_{2} \mathrm{O}}$ are the bulk silica and bulk water electron densities, and $A_{\mathrm{g}}$ is the total external strut surface area. The dependence on relative pressure in eqn (7) is considered via $\phi_{\mathrm{H}_{2} \mathrm{O}}\left(p / p_{0}\right)$, the volume fraction of adsorbed liquid-like water.

The scattering intensity from the micropores within the walls of the mesopores is given by

$$
I_{\mu}\left(q, p / p_{0}\right)=8 \pi^{3} V_{\mathrm{G}} K\left(p / p_{0}\right)\left(1-\phi_{\mathrm{m}}\right) \phi_{\mu}\left(1-\phi_{\mu}\right) f_{\mu}^{2}\left(q, r_{\mu}, \sigma_{\mu}\right) .
$$

This expression is similar to the one reported by Gommes et $a .^{10}$ except for the fact that we choose the micropore form factor $f_{\mu}$ to result from normal distributed spherical micropores with mean radius $r_{\mu}$ and standard deviation $\sigma_{\mu}$. More details on the micropore form factor are given in the ESI. $\dagger$ The only pressure dependent term in eqn (8) is the effective scattering contrast $K=(\Delta \rho)_{\mu}{ }^{2}$. If the micropore filling fraction $\Upsilon_{\mu}\left(p / p_{0}\right)$ is known as a function of relative pressure, the micropore scattering contrast can be simply expressed by

$K\left(p / p_{0}\right)=\left(1-\Upsilon_{\mu}\left(p / p_{0}\right)\right)\left(\rho_{\mathrm{SiO}_{2}}\right)^{2}+\Upsilon_{\mu}\left(p / p_{0}\right)\left(\rho_{\mathrm{SiO}_{2}}-\rho_{\mathrm{H}_{2} \mathrm{O}}\right)^{2}$ 
We note that in the hierarchical pore model presented here, the corona describing the rough surface of the mesopores is not included in the micropore scattering, but in the mesopore term $I_{\mathrm{m}}(q)$ in eqn (6) as described in the following.

$$
\frac{I_{\mathrm{M}}(0)}{I_{\mathrm{M}}\left(p / p_{0}\right)}=\frac{\rho_{\mathrm{SiO}_{2}}{ }^{2}\left(1-\phi_{\mu}\right)^{2}\left(1-\phi_{\mathrm{m}}\right)^{2}}{\left(\rho_{\mathrm{SiO}_{2}}\left(1-\phi_{\mu}\right)\left(1-\phi_{\mathrm{m}}\right)+\rho_{\mathrm{H}_{2} \mathrm{O}}\left(1-\left(1-\phi_{\mu}\right)\left(1-\phi_{\mathrm{m}}\right)\right) x\left(p / p_{0}\right)\right)^{2}}
$$

The mesopore contribution to the SAXS intensity, $I_{\mathrm{m}}(q)$, is essentially the one outlined in Section 4.1 (eqn (2)-(5)). In depth analysis of the SAXS data reveals that the Bragg peak intensities of the investigated sample can be well depicted by eqn (2) along the whole adsorption isotherm, with $\alpha=\alpha\left(p / p_{0}\right)$ describing the successive filling of the corona with liquid-like water until capillary condensation takes place. In other words, there seems to be no distinct film of liquid-like water growing at the mesopore surface. The attempt to employ a form factor which includes a liquid-like film on top of the corona (threestep model $)^{12}$ did not result in improved fit quality compared to a model, where only the relative corona density $\alpha$ was varied and a liquid film covering the mesopore walls was omitted (see Fig. S5 and S6, ESI $\dagger$ ). This suggests a different pore filling mechanism than usual for water in highly corrugated silica mesopores, a hypothesis which is also supported by the quite different shape of the water adsorption isotherm as compared to the nitrogen adsorption isotherm (Fig. S1, ESI $\dagger$ ). intensity can be analyzed. Denoting $x\left(p / p_{0}\right)$ the water filling fraction of the micro- and mesopores, the ratio of eqn (7) for the empty sample $(x=0)$ and for the sample containing the water fraction $x$ at a relative pressure $p / p_{0}$ reads

$$
\frac{I_{\mu}(0)}{I_{\mu}\left(p / p_{0}\right)}=\frac{\rho_{\mathrm{SiO}_{2}}{ }^{2}}{\rho_{\mathrm{SiO}_{2}}{ }^{2}(1-x)+\left(\rho_{\mathrm{SiO}_{2}}-\rho_{\mathrm{H}_{2} \mathrm{O}}\right)^{2} x+\rho_{\mathrm{H}_{2} \mathrm{O}}{ }^{2}\left(\frac{1-\left(\phi_{\mu}+\phi_{\mathrm{m}}\right)}{\phi_{\mu}+\phi_{\mathrm{m}}}\right) x(1-x)}
$$

We also note here that the way the scattering of the mesoporous strut is calculated by eqn (5) already includes scattering from the strut surface. However, this contribution does not correspond to the real surface scattering, because in this case the material outside the strut is silica instead of vacuum. Since the (true) surface scattering is already covered by eqn (7), we must correct the result of $I_{\mathrm{m}}(q)$ from eqn (5) by subtracting the corresponding "wrong" surface scattering contribution. Details are given in the ESI, $\dagger$ Section 5 .

Before simulating the SAXS curves using eqn (6), we analyze two limiting cases, i.e., the intensity at large and at small scattering vector lengths. Because the in situ SAXS measurements were not collected in absolute units, only relative differences in

Table 2 Electron densities of silica and bulk water taken from Zickler et al., ${ }^{12}$ specific microporosity $\phi_{\mu}^{\mathrm{N}_{2}}$ determined from nitrogen adsorption and $\phi_{\mu}^{\text {SAXS }}$ obtained from eqn (10), variance of mesopore radii $\sigma_{\text {meso }}$ estimated from the water adsorption isotherm and micropore diameter $2 r_{\mu}$ with variance $\sigma_{2 r_{\mu}}$ obtained from the SAXS pattern of empty samples

\begin{tabular}{lllllll}
\hline $\begin{array}{l}\rho_{\mathrm{SiO}_{2}} \\
\left(\mathrm{e}^{-} \mathrm{nm}^{-3}\right)\end{array}$ & $\begin{array}{l}\rho_{\mathrm{H}_{2} \mathrm{O}} \\
\left(\mathrm{e}^{-} \mathrm{nm}^{-3}\right)\end{array}$ & $\phi_{\mu}^{\mathrm{N}_{2}}$ & $\phi_{\mu}^{\mathrm{SAXS}}$ & $\begin{array}{l}\sigma_{\text {meso }} \\
(\mathrm{nm})\end{array}$ & $\begin{array}{l}2 r_{\mu} \\
(\mathrm{nm})\end{array}$ & $\begin{array}{l}\sigma_{2 r_{\mu}} \\
(\mathrm{nm})\end{array}$ \\
\hline 654 & 333 & 0.06 & 0.056 & 0.3 & 1.1 & 0.28
\end{tabular}

Assuming that the macroporous surface scattering is dominant for the smallest measured $q$-values of the SAXS profiles, the intensity ratio at small $q$ allows to determine the water adsorption isotherm $x\left(p / p_{0}\right)$ by solving eqn (10) if the micro- $\left(\phi_{\mu}\right)$ and mesopore $\left(\phi_{\mathrm{m}}\right)$ volume fractions are known. In particular, for the highest relative pressures measured, all micro- and mesopores are completely filled with water (i.e. $\left.x\left(p / p_{0} \gtrsim 0.95\right) \approx 1\right)$, and $\phi_{\mu}$ can be calculated by solving eqn (10) for $x=1$ and $\phi_{\mathrm{m}}$ taken from Table 1 . The result listed in Table 2 agrees well with the one obtained from nitrogen adsorption. The slightly lower value may be due to the fact that also the corona contributes to the microporosity obtained from the analysis of the adsorption isotherm, while it was attributed to the mesopores here.

In the limiting case of large scattering vectors, the intensity ratio of the reference state (empty sample) with respect to any state of pore filling can be analyzed in terms of a three-phase model (silica, water, and pore space), assuming this region to be dominated by scattering from the micropores (see $\mathrm{ESI} \dagger$ for details):
Solving eqn (11) for $x$ allows reconstructing the adsorption isotherm. Fig. 5 (red curve) shows that the filling fraction $x$ obtained from eqn (11) approximates the water adsorption isotherm determined by vapor adsorption analysis reasonably well.

To model the SAXS curves over the whole pressure range, the volume fraction of water in the micro- and mesopores must be determined as a function of relative pressure. The micropore filling factor $Y_{\mu}\left(p / p_{0}\right)\left(0 \leq Y_{\mu} \leq 1\right)$ is obtained from the water adsorption isotherm using the theoretical Langmuir isotherm (see ESI $\dagger$ ). The Langmuir isotherm was used in previous studies on this material to model adsorption of water and adsorption induced deformation of micropores ${ }^{17,37}$ and is therefore a reasonable choice to approximate $r_{\mu}\left(p / p_{0}\right)$ in eqn (9). The mesopore filling consists of two parts: the filling of the corona, described by the parameter $\alpha\left(p / p_{0}\right)$ (eqn (3)) and capillary condensation described by the filling factor $r_{\mathrm{m}}\left(p / p_{0}\right)\left(0 \leq r_{\mathrm{m}} \leq 1\right)$, describing the fraction of completely filled mesopores during capillary condensation (eqn (S7), ESI $\dagger$ ). Both parameters are derived from the fit of the Bragg reflections using eqn (2) and keeping the radii $R_{1}$ and $R_{2}$ constant at the values given in Table 1 (see Fig. S4, ESI $\dagger$ ). The relative electron density of the corona does not reach its maximum possible value ( $\alpha=0.76$ here, see Fig. S5, ESI $\dagger$ ) for relative pressures below capillary condensation. This indicates 


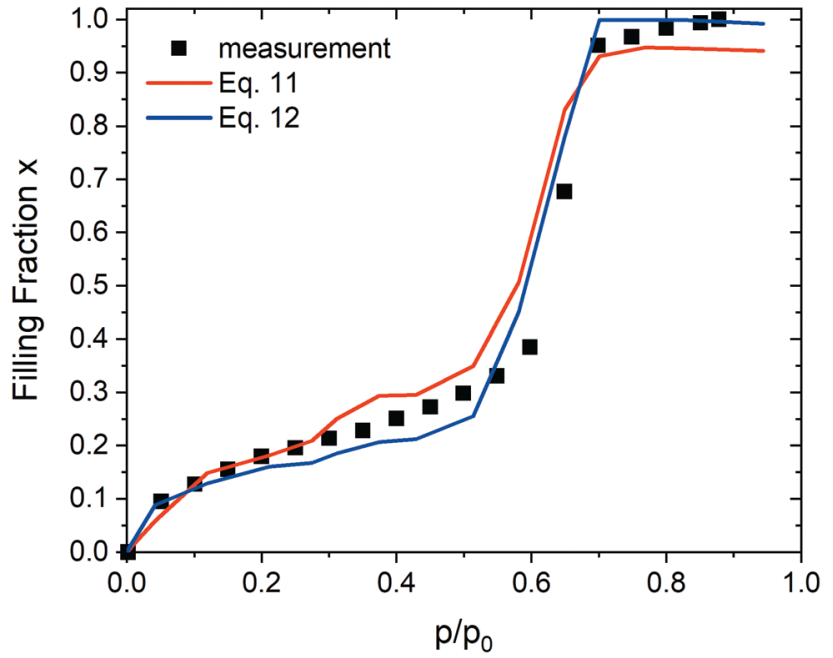

Fig. 5 Pore filling fractions $x$ from SAXS using eqn (11) (red line) and eqn (12) (blue line). The water adsorption isotherm measured independently using a vapor adsorption instrument ${ }^{17}$ is shown by the black symbols.

that the corona is only partially filled and no true multilayer film growth of water occurs at pressures below capillary condensation. For relative pressures above capillary condensation, $\alpha$ reaches the theoretical value for the filled corona in the conducted fits (Fig. S5, ESI $\dagger$ ). For this reason, $\alpha$ was set to its maximum value once capillary condensation occurred and was kept constant for larger pressures.

Given the three parameters $\alpha, \Upsilon_{\mathrm{m}}$ and $\Upsilon_{\mu}$ (Fig. S5, ESI $\dagger$ ), the volume fraction of water at a given relative pressure can easily be calculated

$$
\phi_{\mathrm{H}_{2} \mathrm{O}}=\frac{\frac{\alpha\left(p / p_{0}\right)-\alpha(0)}{\alpha(1)-\alpha(0)} \phi_{\text {corona }}+\Upsilon_{\mathrm{m}}\left(p / p_{0}\right) \phi_{\mathrm{m}, 0}+\Upsilon_{\mu}\left(p / p_{0}\right) \phi_{\mu}}{\phi_{\mathrm{m}}+\phi_{\mu}},
$$

The resulting total pore filling fraction shown in Fig. 5 (blue curve) provides a reasonable accurate description of the adsorption isotherm of the sample.

Given the reasonable agreement of the adsorption isotherm determined from SAXS by eqn (12) with the water adsorption isotherm measured independently, the simulations of the SAXS curves were now performed to quantitatively determine the apparent strain isotherm (see ESI $\dagger$ for more details). The results of these simulations are shown in Fig. 6 for selected relative pressures. The apparent strains extracted from the relative peak shift of the simulated (10) Bragg reflections are depicted in Fig. 7.

\section{Discussion and conclusion}

It is obvious from Fig. 2b, that Model 1, assuming the growth of a liquid-like film on the mesopore surface, is not able to describe the measured apparent strain isotherm. In contrast, the agreement of the simulated apparent strains using Model 2 with the measured ones (see Fig. 7a) is remarkable. In particular, the strong drop of the apparent strain at capillary condensation is well reproduced by the model. In Fig. 7b the resulting strain isotherm after addition of the apparent strain to the SANS strain isotherm by application of eqn (1) is shown. Within experimental accuracy, good agreement is achieved with the experimental SAXS strain isotherm, justifying eqn (1). Fig. 6b and c, showing simulated SAXS patterns for the empty and filled sample, give a qualitative insight for the reason of the strong apparent peak shift at capillary condensation. The micro- and macropore scattering contribute to a considerable amount to the SAXS intensity at the position of the Bragg reflection, thus strongly modifying the diffuse scattering below the peak. Of special note is the crossing point of the macro- and microporous contributions in Fig. 6b and c, which shifts strongly to the right after capillary condensation, introducing a strong change of the slope of the diffuse scattering. This behavior originates in the decrease of the electron density difference of micro- and mesopores upon filling (see eqn (9) and (2) for the respective contributions), and the increase of the difference in electron density of the strut surface scattering with respect to the empty space surrounding it (eqn (7)). Consequently, the scattering intensity of micro- and mesopores decreases and the strut surface scattering increases upon adsorption, which shifts the crossing point of $I_{\mathrm{M}}$ and $I_{\mathrm{m}}+I_{\mu}$ (Fig. $6 \mathrm{~b}$ and c) to larger values of the scattering vector magnitude $q$. This eventually results in an asymmetric broadening of the Bragg peak (see Fig. 6d) resulting in an effective shift of the average peak position to larger $q$ (i.e. to negative apparent strains) due to a considerable reduction of the peak intensity on the left flank of the peak. Since the microand mesopores are completely filled after capillary condensation has occurred, $I_{\mu}$ (eqn (2)), $I_{\mathrm{m}}$ (eqn (9)) and $I_{\mathrm{M}}$ (eqn (7)) remain all constant, which leads to a constant negative apparent strain for $p>p_{\text {cap }}$ in SAXS (Fig. 7a).

In contrast, for pressures below capillary condensation the peak shift to smaller $q$ (positive apparent strain) is supposed to be due to the change of the mesopore formfactor (see inset in Fig. 6d). There is hardly an influence of the macro- and micropore scattering in this region (compare green and black lines in Fig. 7a). Most interestingly, by applying Model 2 the net apparent strain for relative pressures below capillary condensation is positive, as opposed to the application of Model 1 (see Fig. 2b). We recall that in Model 1 a liquid-like water film growing with increasing relative pressure was assumed, while in Model 2 the corona was progressively filled with water without any film growth. Since the micro- and macropore scattering do not influence the peak position markedly in this region, the effect must be due to the mesopore formfactor, which is highly sensitive to the pore filling mechanism. This is a further strong hint that when using water as an adsorbate, the mesopore filling in the present silica material is quite different from the classical picture of homogeneous film growth.

The film thickness derived from structural data derived from SAXS (Tables 1 and 2) and thermodynamic parameters using the DBdB - model (taken from Ludescher et al. ${ }^{17}$ ) indicates that no closed liquid water film exists for $p / p_{0}<0.5$ (see Fig. S2, ESI $\dagger$ ). As the corona represents roughly one third of the total mesopore space (see Table 1), and assuming that the corona is built up by strong surface corrugations and micropores, 
a)

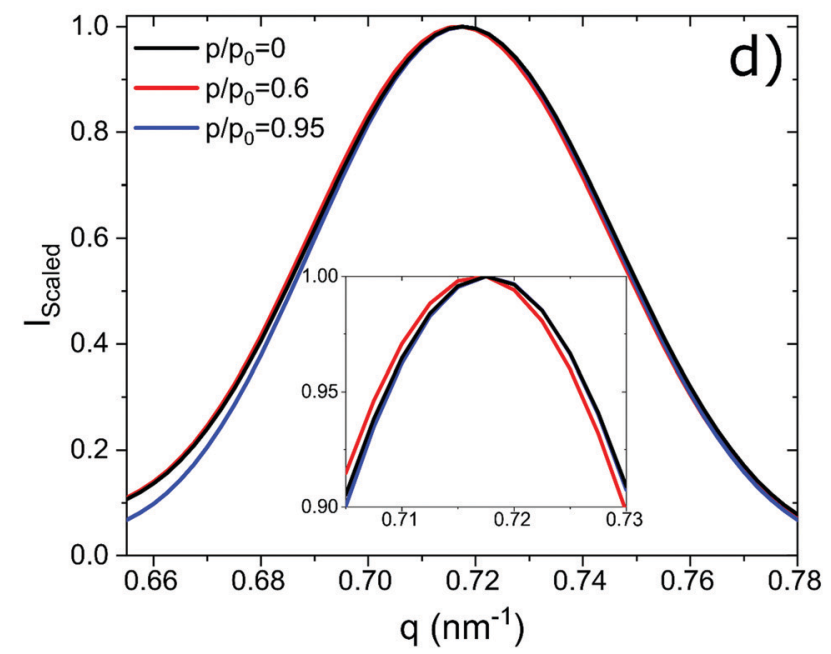
zoom of the peak intensities close to the peak maximum.
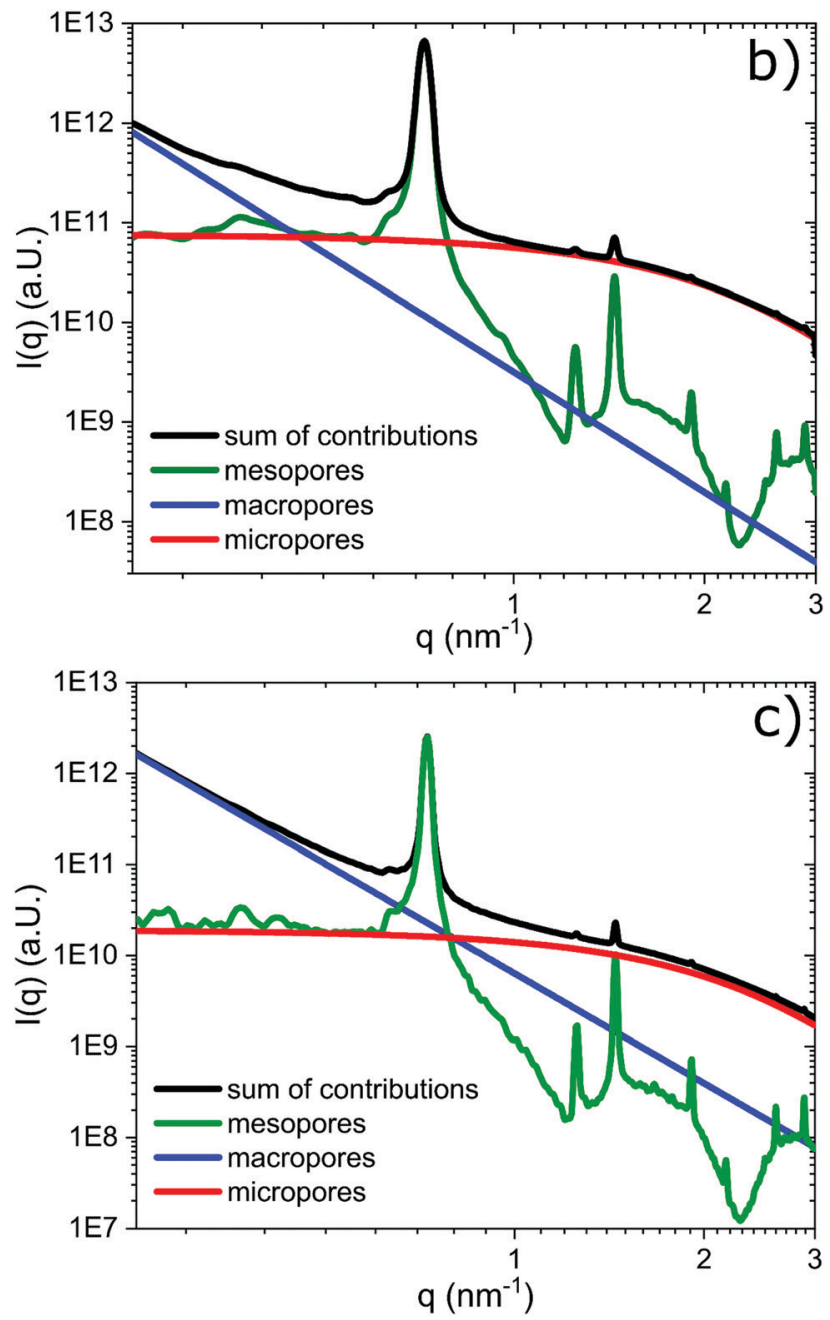

Fig. 6 (a) Electron density profile for Model 2. This model assumes no film formation but gradual filling of the corona until capillary condensation, simulated SAXS profiles using the hierarchical pore model (eqn (6)) are shown for $p / p_{0}=0$ (b) and $p / p_{0}=0.95$ (c) with the individual contributions from macro-, meso-, and micropore scattering highlighted. In (d), the (10) peak scaled to one is shown for three selected relative pressures together with a
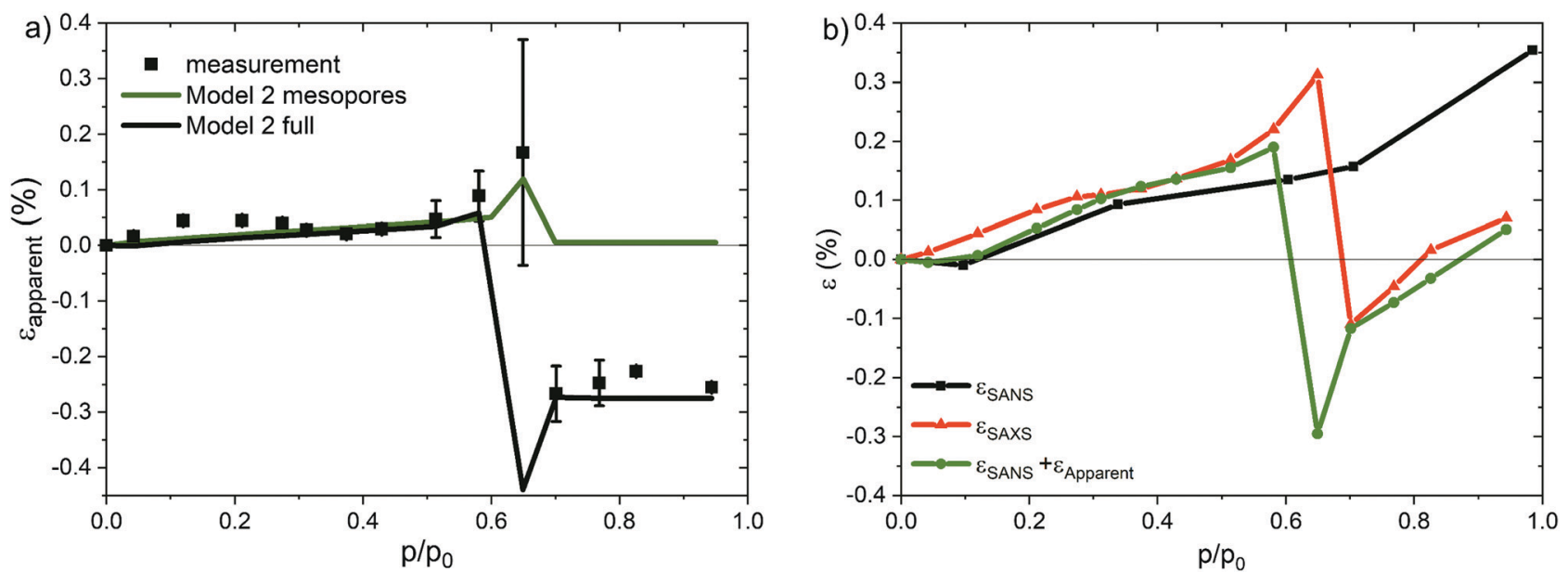

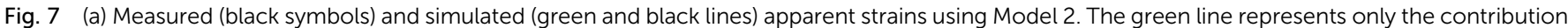

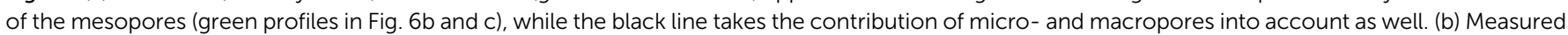

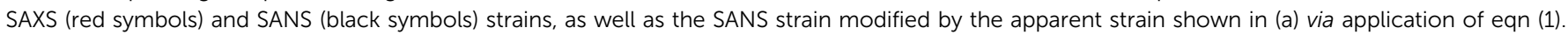


it is not surprising that no pronounced film is discernible in SAXS. Gommes ${ }^{51}$ proposed already several years ago that adsorption in corrugated cylindrical mesopores occurs by filling progressively larger corrugations, until capillary condensation in the mesopores takes place. The effect of this corrugation filling on the position and shape of the hysteresis is not trivial. However, shifts to lower relative pressures of adsorption and evaporation have been demonstrated, ${ }^{51}$ and hence, the adsorption model proposed by Gommes explains tentatively both, the adsorption behavior of water in our material, as well as the discrepancy in mesopore radii between SAXS and nitrogen adsorption (see Fig. S3, ESI $\dagger$ ). However, here we can only give highly indirect evidence for a different adsorption mechanism, and further experiments using different adsorbates will be needed to elucidate the water adsorption mechanism in such materials.

The results from this investigation clarify several points related to adsorption induced deformation in hierarchically nanoporous silica materials using small-angle scattering. First, the difference between the measured strains at low relative pressures between SANS and SAXS show that the apparent strains induced in SAXS mask the contraction of micropores found in Ludescher et al. ${ }^{17}$ for this exact same material. Because subtle effects of the filling of micropores at low relative pressures on SAXS are difficult to model, their correction can only be achieved once the mechanism is well understood. Second, the constant apparent strains at high relative pressures in the filled pore regime show that the concepts introduced by Prass et $a l^{30}$ to extract mechanical properties (the pore load modulus) remain unaffected in SAXS. Yet, higher resolution measurements covering a larger interval in $q$, such as synchrotron X-ray radiation, should help to elucidate more details of the filling mechanism and consequently allow to accurately correct measured strains from apparent effects.

In conclusion, we have developed quantitative models to describe apparent strains by simulation of SAXS data from a hierarchically macro-, meso- and microporous silica material. The SAXS simulations are able to predict apparent strains for a hierarchically porous silica sample quantitatively. In particular, when taking both, the scattering contributions from the ordered mesopores as well as from the micro- and macropores into account, satisfactory correction of strains measured by in situ adsorption SAXS is possible over the whole adsorption isotherm. This is not the case in the capillary condensation regime, however, if only the scattering from the ordered mesopores is considered, due to a strong influence of the diffuse scattering from macro- and micropores. Moreover, there seems to be no film growth of water on the mesopore walls, but rather a continuous filling of strong surface corrugations (corona) at the surface of the mesopore walls. Yet, no real quantitative model for the details of pore filling of these materials with water is available so far.

\section{Conflicts of interest}

There are no conflicts to declare.

\section{Acknowledgements}

We acknowledge financial support from the Austrian Science Foundation FWF (Project I 1605-N20) and the German Science Foundation DFG (Project GZ: RE1148/10-1). This work is based upon experiments performed at the SANS-1 instrument operated by HZG and FRM II at the Heinz Maier-Leibnitz Zentrum (MLZ), Garching, Germany.

\section{Notes and references}

1 F. Chang, J. Zhou, P. Chen, Y. Chen, H. Jia, S. M. I. Saad, Y. Gao, X. Cao and T. Zheng, Asia-Pac. J. Chem. Eng., 2013, 8(4), 618.

2 A. Taguchi and F. Schüth, Microporous Mesoporous Mater., 2005, 77(1), 1.

3 G. Y. Gor, P. Huber and N. Bernstein, Appl. Phys. Rev., 2017, 4(1), 11303.

4 T. Wagner, S. Haffer, C. Weinberger, D. Klaus and M. Tiemann, Chem. Soc. Rev., 2013, 42(9), 4036.

5 M. Thommes, K. Kaneko, A. V. Neimark, J. P. Olivier, F. Rodriguez-Reinoso, J. Rouquerol and K. S. W. Sing, Pure Appl. Chem., 2015, 87(9-10), 1051.

6 K. J. Chao, C. N. Wu, A.-S. Chang and S. F. Hu, Microporous Mesoporous Mater., 1999, 27(2-3), 287.

7 M. Impéror-Clerc, Interface Focus, 2012, 2(5), 589.

8 Scattering Methods and the Properties of Polymer Materials, ed. N. Stribeck and B. Smarsly, Springer Berlin Heidelberg, Berlin, Heidelberg, 2005.

9 B. Smarsly, M. Groenewolt and M. Antonietti, Scattering Methods and the Properties of Polymer Materials, Springer Berlin Heidelberg, Berlin, Heidelberg, 2005, pp. 105-113.

10 C. J. Gommes, G. Prieto and P. E. de Jongh, J. Phys. Chem. C, 2016, 120(3), 1488.

11 M. Impéror-Clerc, P. Davidson and A. Davidson, J. Am. Chem. Soc., 2000, 122(48), 11925.

12 G. A. Zickler, S. Jähnert, W. Wagermaier, S. S. Funari, G. H. Findenegg and O. Paris, Phys. Rev. B: Condens. Matter Mater. Phys., 2006, 73(18), 17.

13 G. Reichenauer, Structural Characterization of Aerogels, in Aerogels Handbook, ed. M. A. Aegerter, N. Leventis and M. M. Koebel, Springer New York, New York, NY, 2011, pp. 449-498.

14 M. Erko, D. Wallacher, A. Brandt and O. Paris, J. Appl. Crystallogr., 2010, 43(1), 1.

15 C. Prehal, D. Weingarth, E. Perre, R. T. Lechner, H. Amenitsch, O. Paris and V. Presser, Energy Environ. Sci., 2015, 8(6), 1725.

16 R. Morak, S. Braxmeier, L. Ludescher, F. Putz, S. Busch, N. Hüsing, G. Reichenauer and O. Paris, J. Appl. Crystallogr., 2017, 50(Pt 5), 1404.

17 L. Ludescher, R. Morak, C. Balzer, A. M. Waag, S. Braxmeier, F. Putz, S. Busch, G. Y. Gor, A. V. Neimark, N. Huesing, G. Reichenauer and O. Paris, Langmuir, 2019, 35(35), 11590.

18 G. Reichenauer and G. W. Scherer, J. Non-Cryst. Solids, 2000, $277(2-3), 162$.

19 G. Reichenauer and G. W. Scherer, J. Non-Cryst. Solids, 2001, 285(1-3), 167. 
20 J. W. McBain and J. Ferguson, J. Phys. Chem., 1927, 31(4), 564. 21 D. H. Bangham and N. Fakhoury, Nature, 1928, 122(3079), 681. 22 M. Erko, D. Wallacher and O. Paris, Appl. Phys. Lett., 2012, 101(18), 181905.

23 J. M. Black, G. Feng, P. F. Fulvio, P. C. Hillesheim, S. Dai, Y. Gogotsi, P. T. Cummings, S. V. Kalinin and N. Balke, Adv. Energy Mater., 2014, 4(3), 1300683.

24 M. M. Hantel, V. Presser, R. Kötz and Y. Gogotsi, Electrochem. Commun., 2011, 13(11), 1221.

25 M. M. Hantel, D. Weingarth and R. Kötz, Carbon, 2014, 69, 275.

26 F. Kaasik, T. Tamm, M. M. Hantel, E. Perre, A. Aabloo, E. Lust, M. Z. Bazant and V. Presser, Electrochem. Commun., 2013, 34, 196.

27 C. Koczwara, S. Rumswinkel, C. Prehal, N. Jäckel, M. S. Elsässer, H. Amenitsch, V. Presser, N. Hüsing and O. Paris, ACS Appl. Mater. Interfaces, 2017, 9(28), 23319.

28 G. A. Zickler, S. Jähnert, S. S. Funari, G. H. Findenegg and O. Paris, J. Appl. Crystallogr., 2007, 40(s1), s522-s526.

29 D. Bellet and G. Dolino, Phys. Rev. B: Condens. Matter Mater. Phys., 1994, 50(23), 17162.

30 J. Prass, D. Müter, P. Fratzl and O. Paris, Appl. Phys. Lett., 2009, 95(8), 83121.

31 G. W. Scherer, J. Am. Ceram. Soc., 1986, 69(6), 473.

32 P. I. Ravikovitch and A. V. Neimark, Langmuir, 2006, 22(26), 11171.

33 G. Y. Gor and A. V. Neimark, Langmuir, 2011, 27(11), 6926.

34 C. Balzer, A. M. Waag, S. Gehret, G. Reichenauer, F. Putz, N. Hüsing, O. Paris, N. Bernstein, G. Y. Gor and A. V. Neimark, Langmuir, 2017, 33(22), 5592.

35 R. Salazar and L. D. Gelb, Mol. Phys., 2004, 102(9-10), 1015.
36 A. L. Kolesnikov, N. Georgi, Y. A. Budkov, J. Möllmer, J. Hofmann, J. Adolphs and R. Gläser, Langmuir, 2018, 34(25), 7575.

37 C. Balzer, A. M. Waag, F. Putz, N. Huesing, O. Paris, G. Y. Gor, A. V. Neimark and G. Reichenauer, Langmuir, 2019, 35(8), 2948.

38 D. Zhao, J. Feng, Q. Huo, N. Melosh, G. H. Fredrickson, B. F. Chmelka and G. D. Stucky, Science, 1998, 279(5350), 548.

39 G. Günther, J. Prass, O. Paris and M. Schoen, Phys. Rev. Lett., 2008, $101(8), 86104$.

40 C. Balzer, R. Morak, M. Erko, C. Triantafillidis, N. Hüsing, G. Reichenauer and O. Paris, Z. Phys. Chem., 2015, 229(7-8), 526.

41 C. T. Kresge, M. E. Leonowicz, W. J. Roth, J. C. Vartuli and J. S. Beck, Nature, 1992, 359(6397), 710.

42 J. Prass, D. Müter, M. Erko and O. Paris, J. Appl. Crystallogr., 2012, 45(4), 798.

43 F. Putz, A. Waag, C. Balzer, S. Braxmeier, M. S. Elsaesser, L. Ludescher, O. Paris, W. J. Malfait, G. Reichenauer and N. Hüsing, Microporous Mesoporous Mater., 2019, 288, 109578.

44 A. Heinemann and S. Mühlbauer, Journal of Large-Scale Research Facilities, 2015, 1(A10), DOI: 10.17815/jlsrf-1-32.

45 S. Jähnert, D. Müter, J. Prass, G. A. Zickler, O. Paris and G. H. Findenegg, J. Phys. Chem. C, 2009, 113(34), 15201.

46 P. Debye, Ann. Phys., 1915, 351(6), 809.

47 J. Broekhoff, J. Catal., 1967, 9(1), 8.

48 B. Derjaguin, Prog. Surf. Sci., 1992, 40(1-4), 46.

49 G. Y. Gor, O. Paris, J. Prass, P. A. Russo, M. M. L. Ribeiro Carrott and A. V. Neimark, Langmuir, 2013, 29(27), 8601.

50 D. Müter, S. Jähnert, J. W. C. Dunlop, G. H. Findenegg and O. Paris, J. Phys. Chem. C, 2009, $113(34), 15211$.

51 C. J. Gommes, Langmuir, 2012, 28(11), 5101. 